\title{
Strong GeV emission accompanying TeV blazar H1426+428
}

\author{
Y. Z. $\operatorname{Fan}^{1,2,3}$, Z. G. Dai ${ }^{1}$, and D. M. Wei ${ }^{2,3}$ \\ 1 Department of Astronomy, Nanjing University, Nanjing 210093, PR China \\ 2 Purple Mountain Observatory, Chinese Academy of Sciences, Nanjing 210008, PR China \\ ${ }^{3}$ National Astronomical Observatories, Chinese Academy of Sciences, Beijing 100012, PR China
}

Received 8 September 2003 / Accepted 28 October 2003

\begin{abstract}
For High frequency BL Lac objects (HBLs) like H1426+428, a significant fraction of their TeV gamma-rays emitted are likely to be absorbed in interactions with the diffuse IR background, yielding $\mathrm{e}^{ \pm}$pairs. The resulting $\mathrm{e}^{ \pm}$pairs generate one hitherto undiscovered $\mathrm{GeV}$ emission by inverse Compton scattering with the cosmic microwave background photons (CMBPs). We study such emission by taking the 1998-2000 CAT data, the reanalyzed 1999 \& 2000 HEGRA data and the corresponding intrinsic spectra proposed by Aharonian et al. (2003a). We numerically calculate the scattered photon spectra for different intergalactic magnetic field (IGMF) strengths. If the IGMF is about $10^{-18} \mathrm{G}$ or weaker, there comes very strong $\mathrm{GeV}$ emission, whose flux is far above the detection sensitivity of the upcoming satellite GLAST! Considered its relatively high redshift $(z=0.129)$, the detected $\mathrm{GeV}$ emission in turn provides us a valuable chance to calibrate the poorly known spectral energy distribution of the intergalactic infrared background, or provides us some reliable constraints on the poorly known IGMF strength.
\end{abstract}

Key words. BL Lacertae objects: general - BL Lacertae objects: individual: H1426+428 - cosmology: diffuse radiation gamma rays: theory

\section{Introduction}

Several High frequency BL Lac objects (HBLs) such as Mkn501, Mkn421, PKS 2155-304, 1ES 2344+514, $\mathrm{H} 1426+428$ and $1 \mathrm{ES} 1959+650$ are of great interest, since they emit TeV photons (Catanese \& Weekes 1999; Aharonian et al. 1999, 2002a; Horns 2002). As early as in 1960s, it was pointed out that the observed high energy gamma photon spectra from $\mathrm{TeV}$ sources might be significantly modified - as the original high energy gamma-rays satisfying $E_{\gamma} E_{\mathrm{B}}>2\left(m_{\mathrm{e}} c^{2}\right)^{2}$ (where $E_{\gamma}$ is the energy of the seed high energy gamma-ray, $E_{\mathrm{B}}$ is the infrared-UV background photon) travel through the intergalactic infrared-UV background, a significant fraction of them will be absorbed, leading to $\mathrm{e}^{ \pm}$pairs (Nikishov 1962; Gould \& Schréder 1965; see also Stecker et al. 1992). If the optical depth to $\mathrm{TeV}$ photons is evaluated, the intrinsic spectrum can be derived. Such calculations were firstly made for Mkn501 (during the 1997 flaring activity), and then for H1426+428, indicating that their intrinsic hight-energy spectra have broad, flat peaks which are much higher than the observed one in the $\sim 5-10 \mathrm{TeV}$ range (e.g. Konopelko et al. 1999; Aharonian et al. 2002; de Jager \& Stecker 2002; Aharonian et al. 2003a). Very recently, Dai et al. (2002) have suggested that inverse Compton (IC) scattering of the resulting $\mathrm{e}^{ \pm}$pairs against the cosmic microwave background photons (CMBPs) may produce

Send offprint requests to: Y. Z. Fan, e-mail: yzfan@pmo. ac.cn a new $\mathrm{GeV}$ emission component in $\mathrm{TeV}$ blazars ${ }^{1}$. As an illustration, they have calculated the well-studied blazar Mkn501, and obtained strong $\mathrm{GeV}$ emission, as long as the intergalactic magnetic field (IGMF) is weak enough.

In this paper, we turn to investigate another important extragalactic object, $\mathrm{H} 1426+428$, which has been detected by Remillard et al. (1989) firstly. With the relatively high redshift $z=0.129$, the optical depth exceeds unity for energies of $\sim 300 \mathrm{GeV}$ (e.g. de Jager \& Stecker 2002). Any detection of a signal at $\mathrm{TeV}$ energies translates directly into a high luminosity of the source. The detection and spectral measurement of H1426+428 by the CAT, and VERITAS groups (Djannati-Ataï et al. 2002; Petry et al. 2002) indicate a steep spectrum between $250 \mathrm{GeV}$ and $1 \mathrm{TeV}$, whereas at higher

\footnotetext{
${ }^{1}$ For Gamma-ray bursts, such Compton scattering leads to an observable, delayed $\mathrm{MeV}-\mathrm{GeV}$ emission component. In fact, there are more than six events have been detected, the well known one is the GRB 940217, whose delayed MeV-GeV emission lasts for $\sim 5000 \mathrm{~s}$ (Hurley et al. 1994). If the model proposed by Plaga (1995) and Cheng \& Cheng (1996) (see also Dai \& Lu 2002; Wang et al. 2003) is correct, the intergalactic magnetic field is $\approx 10^{-20} \mathrm{G}$ (For GRB 940217 , the redshift is unavailable. But with the empirical relation established based on several GRBs with known redshifts, a rough estimate suggests its redshift is $\sim 0.68$ (Fenimore \& Rumirez-Ruiz 2000). On the other hand, the typical energy of the observed delayed photons is $50 \mathrm{MeV}$, which implies the typical energy for the high energy seed photons $E_{\gamma} \approx 0.3 \mathrm{TeV}$. Substituting these two estimates into Eq. (3), we have $B_{\mathrm{IG}} \sim 10^{-20} \mathrm{G}$ ).
} 
energies, detections have been reported by the HEGRA group based upon observations carried out in 1999 and 2000 (Aharonian et al. 2002b). Here, following Dai et al. (2002), we study such hitherto undiscovered $\mathrm{GeV}$ emission by taking the 1998-2000 CAT data (Djannati-Ataï et al. 2002), the reanalyzed $1999 \& 2000$ HEGRA data as well as the derived intrinsic spectra proposed by Aharonian et al. (2003a).

The predicted GeV emission is sensitive to IGMF (Dai et al. 2002). If the IGMF is stronger than $10^{-12} \mathrm{G}$, the formation of a very extended electron/positron Halo $(R \sim 10 \mathrm{Mpc}$ for the primary photons with a typical energy $\sim 1 \mathrm{TeV}$ ) is unavoidable (Aharonian et al. 1994), and its emission is nearly isotropic. If this is the case, the $\mathrm{GeV}$ emission predicted below will be significantly lowered. However, the strength of IGMF has not been determined so far. Faraday rotation measures imply an upper limit of $\sim 10^{-9} \mathrm{G}$ for a field with $1 \mathrm{Mpc}$ correlation length (see Kronberg 1994 for a review). In dynamo theories, to interpret the observed $\mu \mathrm{G}$ magnetic fields in galaxies and X-ray clusters, the seed fields required may be as low as $10^{-20} \mathrm{G}$ (see Kulsrud 1999 for a review). Theoretical calculations of primordial magnetic fields generated during the cosmological QCD or electroweak phase transition show that these fields could be of order $10^{-20} \mathrm{G}$ or even as low as $10^{-29} \mathrm{G}$ (Sigl et al. 1997). The model proposed by Aharonian et al. (2002) to explain the observed TeV emission of Mkn501 suggests that $B_{\mathrm{IG}} \leq 10^{-18} \mathrm{G}$. Modeling the long delayed $\mathrm{MeV}-\mathrm{GeV}$ emission of GRB 940217 suggests that $B_{\mathrm{IG}} \sim 10^{-20} \mathrm{G}$ (see footnote 1). It is evident that all of these claims are far from conclusive. Therefore, observing a hitherto undiscovered $\mathrm{GeV}$ emission component from flares of TeV blazars such as H1426+428, one may be able to obtain important information or constraints on the poorly known IGMFs.

This paper is structured as follows: in Sect. 2, we describe our physical model briefly. In Sect. 3 we provide our numerical results. In Sect. 4 we give our conclusions.

\section{Physical model}

As mentioned above, as the $\gamma$-rays with the energy high up to $\sim 1 \mathrm{TeV}$ travel toward the observer, a significant fraction of them is likely to be absorbed, yielding $\mathrm{e}^{ \pm}$. The pair production optical depth $\tau_{\gamma \gamma}^{\text {ex }}$ depends strongly on the $\gamma$-ray energy ${ }^{2}$ $\left(E_{\gamma}^{\prime} \equiv(1+z) E_{\gamma, \mathrm{obs}}\right)$ and the redshift $(z)$ (throughout this paper, the subscripts "obs" and "loc" denote the parameters measured in the observer and source frames respectively). If a primary photon with energy $E_{\gamma}^{\prime}$ has been absorbed, the resulting $\mathrm{e}^{ \pm}$pairs have Lorentz factors $\left.\gamma_{\mathrm{e}} \simeq E_{\gamma}^{\prime} /\left(2 m_{\mathrm{e}} c^{2}\right) \approx 10^{6} E_{\gamma}^{\prime} / 1 \mathrm{TeV}\right)$, where $m_{\mathrm{e}}$ is the electron mass. Such ultra-relativistic $\mathrm{e}^{ \pm}$pairs will subsequently Compton scatter on the ambient CMBPs, and boost them to an average value $\sim \gamma_{\mathrm{e}}^{2} \bar{\epsilon} \simeq 0.63(1+z)\left(E_{\gamma}^{\prime} / 1 \mathrm{TeV}\right)^{2} \mathrm{GeV}$, where $\bar{\epsilon}=2.7 k T$ is the mean energy of the CMB photons with $T \simeq 2.73(1+z) \mathrm{K}$ and $k$ is the Boltzmann constant.

\footnotetext{
${ }^{2}$ In previous work (e.g. Dai et al. 2002), the red-shift correction of $\gamma_{\mathrm{e}}$ as well as the four timescales involved (see below) has been ignored. However, in the present case, for the relatively high red-shift of $\mathrm{H} 1426+428, z=0.129$, the red-shift correction must be taken into account.
}

Since the emission of blazars is jet-like rather than isotropic, so do the scattered CMBPs. Thus it is necessary to investigate whether significant part of them has been scattered out of the line of sight (the "escaping" effect), or not. For the IC-scattered CMBPs, which move nearly along the path of the ultra-relativistic electrons and the deflection angle is $\sim 1 / \gamma_{\mathrm{e}}$, much less than the typical open angles of blazars. On the other hand, the recoiling of the seed electrons is small, too. It is easy to show that for one scattering, the deflection angle is $<\bar{\epsilon} / m_{\mathrm{e}} c^{2}$. For a seed electron scattering $N$ times, its final deflection angle can be estimated by $\theta_{D} \sim \sqrt{N} \bar{\epsilon} / m_{\mathrm{e}} c^{2}$. Typically, for the IC scattering considered in this paper, $N \approx 1000$, which implies $\theta_{\mathrm{D}} \sim 10^{-7}$. Therefore, the "escaping" effect can be ignored.

\subsection{The GeV emission duration}

As shown in Dai et al. (2002), there are four timescales involved in the emission process: the first is $t_{\mathrm{var}, \mathrm{obs}}$, the observed variability time of the source emission, which has been mentioned earlier.

The second is the well-known angular spreading time

$\Delta t_{\mathrm{A}, \mathrm{obs}} \approx(1+z) \frac{R_{\mathrm{pair}}}{2 \gamma_{\mathrm{e}}^{2} c}=960(1+z)\left(\frac{\gamma_{\mathrm{e}}}{10^{6}}\right)^{-2}\left(\frac{n_{\mathrm{IR}}}{0.1 \mathrm{~cm}^{-3}}\right)^{-1} \mathrm{~s},(1)$

where $R_{\text {pair }}=\left(0.26 \sigma_{\mathrm{T}} n_{\mathrm{IR}}\right)^{-1} \approx 5.8 \times 10^{25}\left(\frac{n_{\mathrm{R}}}{0.1 \mathrm{~cm}^{-3}}\right)^{-1} \mathrm{~cm}$ is the typical pair-production distance, $\sigma_{T}$ is the Thomson cross section, and $n_{\mathrm{IR}} \simeq 0.1 \mathrm{~cm}^{-3}$ is the intergalactic infrared photon number density (Dai \& Lu 2002; Dai et al. 2002; Wang et al. 2003).

The third is the IC cooling timescale. In the absence of any IGMF, the IC cooling timescale in the observer frame would be (Dai et al. 2002)

$\Delta t_{\mathrm{IC}, \mathrm{obs}} \simeq(1+z) t_{\mathrm{IC}, \mathrm{loc}} /\left(2 \gamma_{\mathrm{e}}^{2}\right)=38(1+z)^{-3}\left(\gamma_{\mathrm{e}} / 10^{6}\right)^{-3} \mathrm{~s}$.

$t_{\mathrm{IC}, \mathrm{loc}}$, the IC cooling time scale measured in the source frame is estimated by $t_{\mathrm{IC}, \mathrm{loc}}=3 m_{\mathrm{e}} c /\left(4 \gamma_{\mathrm{e}} \sigma_{T} u_{\mathrm{cmb}}\right)=7.7 \times 10^{13}(1+$ $z)^{-4}\left(\gamma_{\mathrm{e}} / 10^{6}\right)^{-1} \mathrm{~s}$, where $u_{\mathrm{cmb}}=a T^{4}$ is the CMB energy density, and $a$ is the radiation constant.

The fourth is the magnetic deflection time, which appears in the presence of IGMFs. The additional emission time due to magnetic deflection is (Plaga 1995; Dai et al. 2002)

$$
\begin{aligned}
\Delta t_{\mathrm{B}, \mathrm{obs}} & \simeq(1 / 2)(1+z) t_{\mathrm{IC}, \mathrm{loc}} \theta_{\mathrm{B}}^{2} \\
& \simeq 6.1 \times 10^{3}\left(\frac{\gamma_{\mathrm{e}}}{10^{6}}\right)^{-5}\left(\frac{B_{\mathrm{IG}}}{10^{-20} \mathrm{G}}\right)^{2}(1+z)^{-11} \mathrm{~s},
\end{aligned}
$$

for $\theta_{\mathrm{B}}$, the deflection angle $\simeq \lambda_{\mathrm{IC}} / R_{\mathrm{L}}=1.3 \times$ $10^{-5}\left(\gamma_{\mathrm{e}} / 10^{6}\right)^{-2}\left(B_{\mathrm{IG}} / 10^{-20} \mathrm{G}\right)(1+z)^{-4} \ll 1$, where $\lambda_{\mathrm{IC}} \simeq c t_{\mathrm{IC}, \mathrm{loc}}$, $R_{\mathrm{L}}=\gamma_{\mathrm{e}} m_{\mathrm{e}} c^{2} / e B_{\mathrm{IG}}$ is the Larmor radius of the electrons, $B_{\mathrm{IG}}$ is the strength of the IGMFs.

Consequently, the duration estimate of the IC emission from electron/positron pairs scattering off the CMB is $\Delta t\left(\gamma_{\mathrm{e}}\right)=$ $\max \left(\Delta t_{\mathrm{IC}, \mathrm{obs}}, \Delta t_{\mathrm{A}, \mathrm{obs}}, \Delta t_{\mathrm{B}, \mathrm{obs}}, t_{\mathrm{var}, \mathrm{obs}}\right)$. Generally, the typical duration of $\mathrm{GeV}$ emission is determined by the source activity time and the energy dependent magnetic deflection time. For convenience, we introduce a critical Lorentz factor $\gamma_{\mathrm{e}, \mathrm{c}}$ which is defined by $\Delta t_{\mathrm{B}, \mathrm{obs}}=t_{\mathrm{var}, \mathrm{obs}}$

$\frac{\gamma_{\mathrm{e}, \mathrm{c}}}{10^{6}}=0.59\left(\frac{t_{\mathrm{var}, \mathrm{obs}}}{1 \text { day }}\right)^{-1 / 5}\left(\frac{B_{\mathrm{IG}}}{10^{-20} \mathrm{G}}\right)^{2 / 5}(1+z)^{-11 / 5}$. 
For electrons with Lorentz factor $\gamma_{\mathrm{e}}<\gamma_{\mathrm{e}, \mathrm{c}}$, the magnetic deflection angle is such large that most of the scattered CMBPs can not reach the observer in a finite time $t_{\mathrm{var}, \mathrm{obs}}$, which contribute little to the $\mathrm{GeV}$ emission considered here. Therefore, we take $\gamma_{\mathrm{e}, \mathrm{c}}$ as the lower limit for the integration performed below.

\subsection{Basic formulae for calculation}

For the ultra-relativistic seed electrons with the distribution $\mathrm{d} N_{\mathrm{e}} / \mathrm{d} \gamma_{\mathrm{e}}$, the time-averaged scattered photon spectrum is given by (Blumenthal \& Gould 1970; also see Dai \& Lu 2002; Dai et al. 2002)

$$
\frac{\mathrm{d} N_{\gamma}^{\mathrm{SC}}}{\mathrm{d} E_{\gamma^{\prime}}}=\frac{1}{4 \pi D_{\mathrm{L}}^{2}} \iint\left(\frac{\mathrm{d} N_{\mathrm{e}}}{\mathrm{d} \gamma_{\mathrm{e}}}\right)\left(\frac{\mathrm{d} N_{\gamma_{\mathrm{e}}, \epsilon}}{\mathrm{d} t \mathrm{~d} E_{\gamma^{\prime}}}\right) \mathrm{d} t \mathrm{~d} \gamma_{\mathrm{e}},
$$

where $D_{\mathrm{L}}$ is the luminosity distance to the source, $E_{\gamma^{\prime}}$ is the externally scattered photon energy, $t$ is the time measured in the local rest frame, and (Blumenthal \& Gould 1970)

$$
\begin{aligned}
\frac{\mathrm{d} N_{\gamma_{\mathrm{e}}, \epsilon}}{\mathrm{d} t \mathrm{~d} E_{\gamma^{\prime}}}= & \frac{\pi r_{0}^{2} c}{2 \gamma_{\mathrm{e}}^{4}} \frac{n(\epsilon) \mathrm{d} \epsilon}{\epsilon^{2}}\left[2 E_{\gamma^{\prime}} \ln \left(\frac{E_{\gamma^{\prime}}}{4 \gamma_{\mathrm{e}}^{2} \epsilon}\right)\right. \\
& \left.+E_{\gamma^{\prime}}+4 \gamma_{\mathrm{e}}^{2} \epsilon-E_{\gamma^{\prime}}^{2} / 2 \gamma_{\mathrm{e}}^{2} \epsilon\right]
\end{aligned}
$$

is the spectrum of photons scattered (using the Tompson crosssection formula) by an electron with Lorentz factor of $\gamma_{\mathrm{e}}$ from a segment of the CMBP gas of differential number density (Blumenthal \& Gould 1970)

$n(\epsilon)=\left[\pi^{2}(\hbar c)^{3}\right]^{-1}\left[\epsilon^{2} /\left(\mathrm{e}^{\epsilon / k T}-1\right)\right]$.

The integration for variable $\gamma_{\mathrm{e}}$ ranges from $\gamma_{\mathrm{e}, \mathrm{c}}$ to $E_{\text {up,cut }}^{\prime} / 2 m_{\mathrm{e}} c^{2}$, where $E_{\text {up,cut }}^{\prime}$ is the observed upper cut-off energy timed by $(1+z)$. Please note that this is only valid at the beginning since $\gamma_{\mathrm{e}}$ decreases with $t$, which can be written into $\gamma_{\mathrm{e}(\mathrm{t})}=\gamma_{\mathrm{e}, 0} /\left(1+b \gamma_{\mathrm{e}, 0} t\right)$, where $\gamma_{\mathrm{e}, 0}$ is the original Lorentz factor of the seed $\mathrm{e}^{ \pm}$pairs, $b=4 \sigma_{\mathrm{T}} U_{\mathrm{cmb}} / 3 m_{\mathrm{e}} c$.

Dai \& Lu (2002) simply replace $\int \mathrm{d} t$ by $t_{\mathrm{IC}, \text { loc }}$, and assume the seed electrons have a distribution $\propto \gamma_{\mathrm{e}}^{-\Gamma_{1}}$. With these simplifications, they have shown that in the Thomson limit, the scattered photon spectrum $\propto E_{\gamma^{\prime}}^{-\left(\Gamma_{1}+2\right) / 2}$ for $\Delta t_{\mathrm{B}, \mathrm{obs}}\left(\gamma_{\mathrm{e}}\right) \ll t_{\mathrm{var}, \mathrm{obs}}$ and $\propto E_{\gamma^{\prime}}^{-\left(\Gamma_{1}-3\right) / 2}$ for $\Delta t_{\mathrm{B}, \mathrm{obs}}\left(\gamma_{\mathrm{e}}\right) \gg t_{\mathrm{var}, \mathrm{obs}}$. Instead of taking such a simple estimate, below we make a detailed numerical calculation: the integration for variable $t$ ranges from 0 to $7.7 \times 10^{13}(1+z)^{-4}\left(\gamma_{\mathrm{e}, \mathrm{c}} / 10^{6}\right)^{-1} \mathrm{~s}$.

\section{Numerical result}

In our calculation, the original seed electron energy spectrum is derived as follows: we simply apply a polynomial fit to the local highly absorbed photon spectrum: setting $x \equiv$ $\log \left(E_{\gamma}^{\prime} / 1.0 \mathrm{TeV}\right)$, we have

$\frac{\mathrm{d} N_{\gamma}}{\mathrm{d} E_{\gamma}^{\prime}} \approx 10^{0.79046-3.31624 x+1.15145 x^{2}-0.79715 x^{3}}$

which is in units of $4 \pi D_{\mathrm{L}}^{2} 10^{-12} \mathrm{ph} \mathrm{cm}^{-2} \mathrm{~s}^{-1} \mathrm{TeV}^{-1}$. The local intrinsic photon spectra $\mathrm{d} N_{\gamma, \text { int }}^{\mathrm{i}} / \mathrm{d} E_{\gamma^{\prime}}$ (also in units of

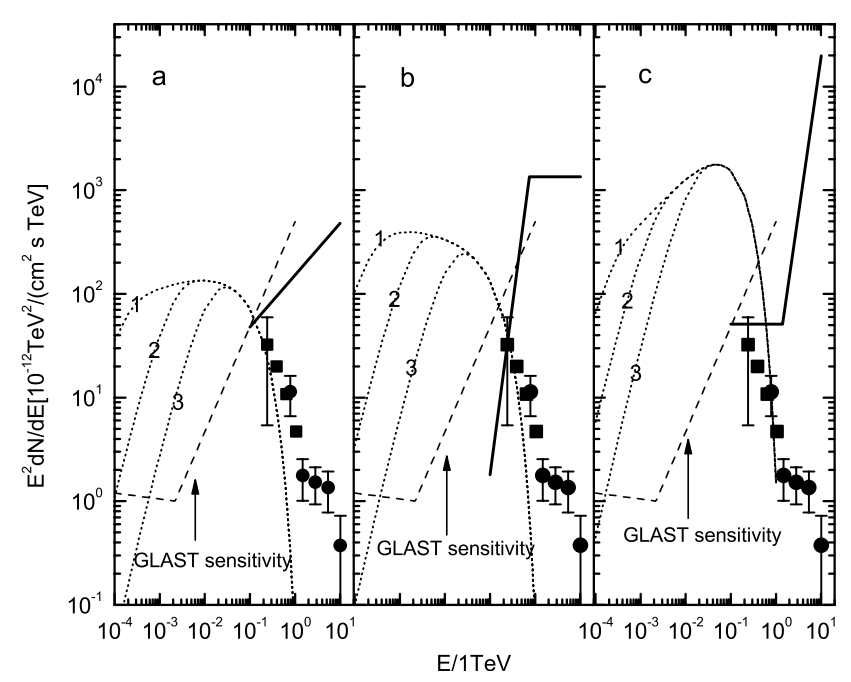

Fig. 1. The time-averaged high energy Gamma-ray spectra of H1426 +428 for a flare with $t_{\text {var,obs }}=2$ days. The filled circles are the reanalyzed 1999/2000 spectral data measured by HEGRA (Aharonian et al. 2003a), and the filled rectangles are the 1998-2000 spectral data measured by the CAT (Djannati-Ataï et al. 2002). In a), b) and c): the thick solid lines represent the derived intrinsic spectra based on the correction model proposed by Primack et al. (2001), Aharonian et al. (2003a) and Malkan \& Stecker (2001) (see also Fig. 4 of Aharonian et al. 2003a) respectively; the thick dashed lines represent the GLAST sensitivity computed for an exposure time of 2 days; the dotted lines are the secondary photon spectra calculated in the text from the resulting $\mathrm{e}^{ \pm}$pairs interacting with the CMBPs, the number $1-3$ correspond to IGMF strength of $10^{-20} \mathrm{G}, 10^{-19} \mathrm{G}$ and $10^{-18} \mathrm{G}$, respectively.

$\left.4 \pi D_{\mathrm{L}}^{2} 10^{-12} \mathrm{ph} \mathrm{cm}^{-2} \mathrm{~s}^{-1} \mathrm{TeV}^{-1}\right)$ can be approximated as follows (Aharonian et al. 2003a):

$\frac{\mathrm{d} N_{\gamma, \text { int }}^{\mathrm{a}}}{\mathrm{d} E_{\gamma}^{\prime}} \approx 5.8 \times 10^{3}\left(\frac{E_{\gamma}^{\prime}}{0.1 \mathrm{TeV}}\right)^{-3 / 2}$,

$\frac{\mathrm{d} N_{\gamma, \text { int }}^{\mathrm{b}}}{\mathrm{d} E_{\gamma}^{\prime}} \approx \begin{cases}1.6 \times 10^{2}\left(\frac{E_{\gamma}^{\prime}}{0.10 \mathrm{TeV}}\right), & E_{\gamma}^{\prime} \leq 0.85 \mathrm{TeV}, \\ 1.4 \times 10^{3}\left(\frac{E_{\gamma}^{\prime}}{0.85 \mathrm{TeV}}\right)^{-2}, & E_{\gamma}^{\prime} \geq 0.85 \mathrm{TeV},\end{cases}$

$\frac{\mathrm{d} N_{\gamma, \text { int }}^{\mathrm{c}}}{\mathrm{d} E_{\gamma}^{\prime}} \approx \begin{cases}6.5 \times 10^{3}\left(\frac{E_{\gamma}^{\prime}}{0.10 \mathrm{TeV}}\right)^{-2}, & E_{\gamma}^{\prime} \leq 1.6 \mathrm{TeV}, \\ 25.4\left(\frac{E_{\gamma}^{\prime}}{1.60 \mathrm{TeV}}\right), & E_{\gamma}^{\prime} \geq 1.6 \mathrm{TeV},\end{cases}$

where the superscript $i=a, b, c$ represent the correction models proposed by Primack et al. (2001), Aharonian et al. (2003a) and Malkan \& Stecker (2001) respectively. Since $E_{\gamma}^{\prime} \approx$ $1\left(\gamma_{e} / 10^{6}\right) \mathrm{TeV}$, we have (including positrons)

$\frac{\mathrm{d} N_{\mathrm{e}}^{\mathrm{i}}}{\mathrm{d} \gamma_{\mathrm{e}}} \approx 2 \times 10^{-6}\left(\frac{\mathrm{d} N_{\gamma, \text { int }}^{\mathrm{i}}}{\mathrm{d} E_{\gamma}^{\prime}}-\frac{\mathrm{d} N_{\gamma}}{\mathrm{d} E_{\gamma}^{\prime}}\right)$.

Now we can calculate the spectra of the scattered CMBPs with Eqs. (5)-(12). Please note that the $\epsilon, E_{\gamma^{\prime}}$ in Eqs. (6), (7) are all measured in the local frame rather than by the observer.

The numerical result has been shown in Fig. 1. As long as the IGMF strength is weak enough, there is the very strong $\mathrm{GeV}$ emission (although it is intrinsic spectrum-dependent), 
whose flux is far above the thick dashed line, the GLAST sensitivity computed for an exposure time of 2 days. Just as shown in Fig. 1, the inferred intrinsic spectra is most sensitive to the characteristics of the extragalactic background light. As a consequence, different correction models lead to much different intrinsic spectra, so does the accompanying $\mathrm{GeV}$ emission, and thus the detection of such emission may in turn provide us a valuable chance to test those IR background absorption models.

In our calculations, as an illustration, we take $E_{\text {up,cut }}^{\prime}=$ $10.2(1+z) \mathrm{TeV}$ (Above which there is no observation available), which is conservative. Even so, with the three correction models taken in Aharonian et al. (2003a), the predicted timeaveraged scattered photon energy flux are far above (case b, c), or at least comparable with (case a) that of the well studied Blazar Mkn501 (see Dai et al. 2002 for detail). One problem arises, just as shown in Fig. 1c, the predicted new hard $\gamma$-ray emission at the energy $\sim 0.24 \mathrm{TeV}$ is about ten times that observed. In principle, such emission may be absorbed by the background IR/UV photons again, but the resulting flux is still high above the observed (for $E_{\gamma} \sim 0.24 \mathrm{TeV}, \tau_{\gamma \gamma}^{\mathrm{ex}} \sim 1$ ). Two possibilities exist: one is that the derived intrinsic spectrum based on the correction model proposed by Malken \& Stecker (2001) is much harder than what it really is. Another is that the IGMF might be strong enough, the observable $\mathrm{GeV}-\mathrm{TeV}$ emission flux has been suppressed significantly.

For Blazar H1426+428, due to the poorly known X-ray band spectrum, we cannot provide a definite SSC spectrum as Dai et al. (2002) have done for Mkn501. Even so, as shown in Fig. 1, at a first glance, the SSC component is likely far below than the externally scattered photon flux.

\section{Conclusions}

The HBL H1426+428 is distinguished by its relatively high redshift as well as its strong $\mathrm{TeV}$ energy emission. In this paper, with the recently observed data as well as the derived intrinsic spectrum, we have studied the possible accompanying $\mathrm{GeV}$ emission, which is due to the IC scattering of CMBPs by the $\mathrm{e}^{ \pm}$ pairs produced in interactions of high energy photons with the cosmic infrared-UV background photons. If the IGMF is weak enough, there is very strong hitherto undiscovered $\mathrm{GeV}-\mathrm{TeV}$ emission, whose flux is far above or at least comparable with that of the well-studied HBLs Mkn501 (Dai et al. 2002). With the upcoming satellite GLAST, planned for launch in 2005, it will be easy to detect such emission directly. We also noted that the shape of the inferred source spectra is most sensitive to the characteristics of the extragalactic background light. A different correction model leads to a different intrinsic spectrum, so does the accompanying $\mathrm{GeV}$ emission (see Fig. 1 for detail). Thus the detection of such emission may in turn provide us a valuable chance to test those IR background absorption models.

In Aharonian et al. (2003b), the detection of $\mathrm{TeV}$ $\gamma$-rays from the BL Lac 1ES1959+650 has been reported. $1 \mathrm{ES} 1959+650$ is located at a redshift of $z=0.047$, providing an intermediate distance between the nearby blazars Mkn421 and Mkn501, and the much more distant object H1426+428. Without doubt, for such a source, the observed $\mathrm{TeV}$ emission has been absorbed significantly by the infrared-UV background photons, and thus a strong $\mathrm{GeV}$ emission is expected, as long as the IGMF is weak enough.

Acknowledgements. We would like to thank Drs Y. F. Huang, X. Y. Wang \& X. F. Wu for valuable comments. We also thank the anonymous referee for her/his useful suggestions that enable us to improve the paper significantly. This work was supported by the National Natural Science Foundation of China (grants 10073022, 10225314 and 10233010), the National 973 Project (NKBRSF G19990754) and the Foundation for the Author of National Excellent Doctoral Dissertation of P. R. China. (Project No: 200125).

\section{References}

Aharonian, F. A., Akhperjanian, A., \& Barrio, J., et al. 1999, A\&A, 349,11

Aharonian, F. A., Akhperjanian, A., \& Beilicke, M., et al. 2002a, A\&A, 393, 89

Aharonian, F. A., Akhperjanian, A., \& Barrio, J., et al. 2002b, A\&A, 384, L23

Aharonian, F. A., Akhperjanian, A., \& Beilicke, M., et al. 2003a, A\&A, 403, 523

Aharonian, F. A., Akhperjanian, A., \& Beilicke, M., et al. 2003b, A\&A, 406, L9

Aharonian, F. A., Coppi, P. S., \& Völk, H. J. 1994, ApJ, 423, L5

Aharonian, F. A., Timokhin, A. N., \& Plyasheshnikov, A. V. 2002, A\&A, 384, 834

Blumenthal, G. R., \& Gould, R. J. 1970, Rev. Mod. Phys., 42, 237

Catanese, M., \& Weekes, T. C. 1999, PASP, 111, 1193

Cheng, L. X., \& Cheng, K. S. 1996, ApJ, 459, L79

Dai, Z. G., \& Lu, T. 2002, ApJ, 580, 1013

Dai, Z. G., Zhang, B., Gou, L. J., Meszaros, P., \& Waxman, E. 2002, ApJ, 580, L7

de Jager, O. C., \& Stecker, F. W. 2002, ApJ, 566, 738

Djannati-Ataï, A., Khelifi, B., Vorobiov, S., et al. 2002, A\&A, 391, L25

Fenimore, E. E., \& Ramirez-Ruiz, E. 2000, ApJ, submitted [astro-ph/0004176]

Hauser, M. G., \& Dwek, E. 2001, ARA\&A, 39, 249

Horns, D. 2002, in the Proceedings for High Energy Blazar astronomy, Turku, Finland 2002 [astro-ph/0209454]

Hurley, K., Dingus, B. L., \& Mukherjee, R., et al. 1994, Nature, 372, 652

Gould, R. J., \& Schréder, G. P. 1967, Phys. Rev., 155, 1408

Katarzyński, K., Sol, H., \& Kus, A. 2001, A\&A, 367, 809

Kino, M., Takahara, F., \& Kusunose, M. 2002, ApJ, 564, 97

Konopelko, A. K., Kirk, J. G., Stecker, F. W., \& Mastichiadis, A. 1999, ApJ, 518, L13

Kronberg, P. P. 1994, Rep. Prog. Phys., 57, 325

Kulsurd, R. 1999, ARA\&A, 37, 37

Malkan, M. A., \& Stecker, F. W. 2001, ApJ, 555, 641

Nikishov, A. I. 1962, Sov. Phys. - JETP, 14, 393

Petry, D., Böttcher, M., \& Connaughton, V., et al. 2000, ApJ, 536, 742

Pian, E., Vacanti, G., \& Tagliaferri, G., et al. 1998, ApJ, 492, L17

Plaga, R. 1995, Nature, 374, 430

Primack, J. R., Sommerville, R. S., Bullock, J. S., \& Devriendt, J. E. G. 2001, in High Energy Gamma-Ray Astronomy: Intern. Symp., AIP Conf. Proc., 558, 463

Remillard, R. A., Tuohy, I. R., Brissenden, R. J. V., et al. 1989, ApJ, 315,140

Sigl, G., Olinto, A. V., \& Jedamzik, K. 1997, Phys. Rev. D., 55, 4582

Stecker, F. W., de Jager, O. C., \& Salamon, F. W. 1992, ApJ, 390, L49

Wang, X. Y., Cheng, K. S., Dai, Z. G., \& Lu, T. 2003, ApJ, submitted 